\title{
Gravity, the origin of the mass in the Cosmos.
}

\author{
F. PAVÍA ${ }^{1,1}$, M. ÁLVAREZ ${ }^{12}$ \\ ${ }^{1}$ Agrupación Astronómica de la Safor, Pellers, 12 - bajo 46702 Gandia, Valencia, \\ España.
}

\footnotetext{
${ }^{1}$ Engineer from the Escuela Técnica Superior del ICAI (ICAI School of Engineering in Higher Education) (Madrid), Masters in Astrophysics Valencian International University,

ORCID-ID: 0000-0002-4055-0707

Email: paco.pavia.alemany@gmail.com
}

${ }^{2}$ Computer scientist, Director of the Federation of Astronomical Associations of Spain, President of the Agrupación Astronómica de la Safor,

ORCID-ID: 0000-0001-8181-8418

Email: maralvilla@gmail.com 


\title{
Gravity, the origin of the mass in the Cosmos.
}

\begin{abstract}
The most accepted theory for the evolution of the Cosmos is the Big Bang theory, which suggests that, at the beginning, the entire mass-energy of the Cosmos was concentrated within an extremely small, dense and hot singularity. Here, we present a new physical formula that, although obtained in an extremely simple way, has significant implications in Cosmology. This equation indicates that the mass of the Cosmos has grown proportionally with time. This growth equates to a Planck mass for each unit of Planck Time, which is, the mass of 200000 suns per second. Finally the total energy of the Cosmos is demonstrated to be zero.
\end{abstract}

Key words: Cosmology, Big Bang, gravity, zero-energy cosmos, change of paradigm.

\section{INTRODUCTION}

At the end of the nineteenth century, most scientists were convinced that in the field of physics, there was little to be done-that all the specialties within the discipline were complete and that, at most, one decimal point could be added to the values of some constants.

It was precisely at the end of the nineteenth century, and the beginning of the twentieth century that the greatest progress and transformation took place in this discipline. Those huge advances were made by Max Planck with its Quantum Theory, and by Albert Einstein with its Theory of Relativity.

At that time, scientists and educated men had an idea of the Universe that was quite generalized and accepted: that of a Static and Eternal Cosmos, that is, without changes and without a beginning or end in time, in addition to being very minute and only constituted by the objects of our own galaxy.

This conception of the Cosmos, Static and Eternal is the one that Albert Einstein defended for a long time. He was so convinced of this hypothesis, that he was forced to introduce "ad hoc" the Cosmological Constant in his General Relativity Equation, to be consistent with the hypothesis of a stable and static universe, despite of the warnings of Friedman (Friedman 1922), Lemaître (Lemaître 1927), and Hubble (Hubble 1929) and others, who indicated the possibility of an expanding Universe, to which he always responded with harsh tones.

When Edwin Hubble demonstrated that the Cosmos is expanding (Hubble 1929), the hypothesis of the Static Cosmos ceased to make sense. However, Einstein was still convinced that this hypothesis was true. When Einstein visited Hubble in 1931 at the Mount Wilson Observatory, which had the world's most powerful 254-centimeter telescope (Walter Isaacson 2008), and saw the evidence presented by Edwin, he had to admit that he «had made the greatest mistake of his life» by inserting the Cosmological Constant into his equation of General Relativity (Gamow G. 1970).

Several researchers saw the need to replace the incorrect hypothesis with a new one, which, despite of the expansion of the Cosmos, would translate into minimum changes to the original idea. Fred Hoyle and others developed a new theory of a Stationary Cosmos, in which mass is continuously being created to maintain the constant density of the Universe (Hoyle Fred 1948). 
George Gamow proposed another alternative, the Big Bang theory, based on the ideas of the Jesuit priest Lemaitre about the expansion of the universe, and referred to as "the hypothesis of the primeval atom" or the "cosmic egg" (Lemaitre G. 1927). According to this theory, at the beginning, all the mass-energy of the current Cosmos was within an extremely small, dense and hot singularity, and after a kind of explosion, an expansion began, and it actually keeps expanding up until today. In Hoyle's theory, density remains constant, whereas in the Big Bang theories, mass-energy remains constant.

There are three main setbacks in the model: the flatness problem, the horizon problem and the monopole problem.

To overcome the adversities presented by the Big Bang hypothesis, Alan Guth proposed the theory "of the inflationary model", according to which the universe suffered an exponentially accelerated expansion called "the inflation". Inflation is considered to have lasted between $10^{-36}$ and $10^{-35}$ seconds.

Meanwhile, the Radius of the Universe grew from just over Planck's length to approximately one metre (Alan H. Guth 1999).

Guth, in the appendix of his aforementioned publication, also contributed the idea that «energy is released when a gravitational field is created... which should be negative».

Already in 1973 some ideas were presented that defended that the entire Universe could arise from a fluctuation of the vacuum without violating the conservation laws, by compensating the positive energy of the mass with the negative gravitational energy. (Fomin P.I. 1973) and (Tryon E.P. 1973).

Alexander Vilenkin contributed the theory that the universe was created out of nothing (Vilenkin A.1982).

Several authors do not believe in the Big Bang theory as a cosmological model and have proposed alternative theories. Peter Lynds proposed the model of a universe that is repeated an infinite number of times, and as a consequence, time is cyclical (Lynds P. 2007).

We should note that none of the theories that have been mentioned, including the Big Bang theory, relies on a mathematical-physical basis; these are speculative theories that have been more or less credible in their time.

The Big Bang theory with the Inflation is currently accepted by the academic world.

Steven Weinberg in "The first three minutes" shows us how the primitive universe developed in preparation to evolve into the present cosmos (Steven Weinberg 1978).

However, the great question about the origin of the "initial singularity", the "primeval atom" or the "cosmic egg" remains. Lawrence M. Krauss and other authors have proposed the idea that "In quantum gravity, universes can, and indeed always will, spontaneously appear from nothing. Such universes need not be empty, but can have matter and radiation in them, as long as the total energy, including the negative energy associated with gravity, is zero». (Lawrence M. Krauss 2012)

Here we present a new equation that responds to our concerns, providing the new theory of the Cosmos with a mathematical-physical basis, and explaining the origin of mass-energy in the Cosmos, with gravity playing a fundamental role. Moreover, this equation allows us to state that the total energy of the Cosmos is zero. 


\section{DIMENSIONAL EXPRESSION OF MASS}

Based on the following two basic expressions,

$$
\begin{aligned}
& \text { - } \quad \mathrm{F}=G \frac{M m}{D^{2}} \quad \text { expressed dimensionally as } \quad[\mathrm{F}]=\left[\mathrm{G} \mathrm{M}^{2} \mathrm{~L}^{-2}\right] \\
& \text { - } \mathrm{F}=m a \quad \text { expressed dimensionally as }[\mathrm{F}]=\left[\mathrm{M} \mathrm{L} \mathrm{T}^{-2}\right]
\end{aligned}
$$

Equating both expressions: $\quad\left[\mathrm{G} \mathrm{M}^{2} \mathrm{~L}^{-2}\right]=\left[\mathrm{MLT}^{-2}\right]$

By simplifying the Mass term, we obtain:

$$
[\mathrm{M}]=\left[\mathrm{G}^{-1} \mathrm{~L}^{3} \mathrm{~T}^{-2}\right]
$$

To transform this expression into an equivalent expression with an easier interpretation, we can express the length "L" as a function of the speed of light in the vacuum "c", and the time "T" in the following form: $\quad[\mathrm{L}]=[\mathrm{c} \mathrm{T}]$

Obtaining the following expression:

$$
[\mathrm{M}]=\left[\mathrm{c}^{3} \mathrm{G}^{-1} \mathrm{~T}\right]
$$

The constants $[\mathrm{G}]=\left[\mathrm{L}^{3} \mathrm{M}^{-1} \mathrm{~T}^{-2}\right]$ and $[\mathrm{c}]=\left[\mathrm{LT}^{-1}\right]$ can be considered in their double function, either as a kind of dimensional parenthesis within the expression, or as universal constants, as long as the dimensional homogeneity of the expression is preserved.

This is a very simple deductive process that provides us with an extremely simple expression with a profound meaning.

In this expression $[\mathrm{M}]=\left[\mathrm{c}^{3} \mathrm{G}^{-1} \mathrm{~T}\right]$, the mass "M" is dimensionally a function of two universal constants "c" and "G" (dimensional constants with values that depend on the system of units used) and a single variable of time "T".

Expression (4) is not a physical formula because it has been deduced from dimensional equations, which can mask the existence of non-dimensional constants. It can be converted into a physical formula by introducing a dimensionless constant " $\mathrm{K}$ " in the following way:

$$
\mathrm{M}=\mathrm{Kc}^{3} \mathrm{G}^{-1} \mathrm{~T}
$$

Although expression (4) clearly shows that the mass "M" grows proportionally with time, it is necessary to determine the value of the possibly dimensionless constant "K", which when added to the two dimensional constants "c" and "G", transforms expression (4) into a physical equation that allows quantitative results to be obtained.

\section{OBTAINING THE VALUE OF "K" FOR PLANCK TIME}

Directly using the expressions corresponding to the Planck Mass " $\mathrm{M}_{\mathrm{P}}$ " and Planck Time " $\mathrm{T}_{\mathrm{P}}$ " (Sanchez del Rio C. 1997), we obtain the following:

$$
\begin{gathered}
\mathrm{M}_{\mathrm{P}}=\sqrt{\hbar c / G} ; \quad \mathrm{T}_{\mathrm{P}}=\sqrt{\hbar G / c^{5}} \\
\left(\mathrm{M}_{\mathrm{P}}\right)^{2} /\left(\mathrm{T}_{\mathrm{P}}\right)^{2}=\mathrm{c}^{6} / \mathrm{G}^{2} \\
\mathrm{M}_{\mathrm{P}}=\mathrm{c}^{3} \mathrm{G}^{-1} \mathrm{~T}_{\mathrm{P}}
\end{gathered}
$$


For the particular case of the Planck Time, we verify that the dimensional expression of mass (4) corresponds with formula (5), which was obtained from the definition of the Planck units, i.e., the value of $\mathrm{K}$ is the unit.

The correspondence between (4) and (5) shows that expression,

$$
\mathrm{M}=\mathrm{c}^{3} \mathrm{G}^{-1} \mathrm{~T}
$$

represents the correct physical equation.

(Five different ways of obtaining this formula are shown in Appendix A)

(Appendix B shows the calculations of Dr. Dimitar, who obtained a similar formula but did not realize its true meaning).

\section{INTERPRETATION OF THE EQUATION $M=c^{3} \mathrm{G}^{-1} \mathrm{~T}$}

The interpretation of equation (6) provides us with a very different conception of the Cosmos from the established one.

1. $\quad$ For $\mathrm{T}=0$, the mass $\mathrm{M}=0$.

2. The mass grows proportionally with time. Therefore, we have the history of the mass of the Cosmos.

3. The relationship between the universal constants $" \mathrm{c}^{3} / \mathrm{G}$ " seems to be generating the mass.

\section{DETERMINATION OF THE GROWTH RATE OF THE MASS OF THE COSMOS}

We can apply equation (6), to the history of the Cosmos. Assuming that the mass of the cosmos grows proportionally with time we can calculate the mass of the Cosmos after the first second of existence and its growth rate per second, $\Delta \mathrm{Ms}^{-1}$, based on the following:

$$
\mathrm{G}=6.674 \times 10^{-11} \mathrm{~m}^{3} \mathrm{~kg}^{-1} \mathrm{~s}^{-2} \quad ; \mathrm{c}=3 \times 10^{8} \mathrm{~m} \mathrm{~s}^{-1}
$$

Substituting equation (6) $\Delta \mathrm{Ms}^{-1}=4 \times 10^{35} \mathrm{kgs}^{-1}$

Assume that the mass of the Sun is $2 \times 10^{30} \mathrm{~kg}$ (Martínez 2005), which corresponds to $\Delta \mathrm{Ms}^{-1}$ $=2 \times 10^{5}$ suns per second.

That is, a mass equivalent to 200000 suns is generated per second.

\section{DETERMINATION OF THE MASS OF THE COSMOS CREATED FROM THE BEGINNING}

The current cosmology estimates the present age as: 13.7 billion years, (The WMAP 2012)

Since the beginning of the Cosmos, the elapsed time in seconds is approximately

$$
4.32 \times 10^{17} \mathrm{~s}
$$

Applying the obtained growth rate, it gives us a total mass of the Cosmos today that is equivalent to

$$
8.64 \times 10^{22} \text { suns }
$$


According to our calculations $8.64 \times 10^{22}$ suns $\times 2 \times 10^{30} \mathrm{~kg}$ per sun $=1.73 \times 10^{53} \mathrm{~kg}$

\section{IS THIS MASS COMPATIBLE WITH OTHER ESTIMATES?}

In a simple, straightforward and unpretentious way:

Assuming $10^{11}$ to $4 \times 10^{11}$ stars in our Galaxy (Maggie Masetti. 2015)

Assuming $2.46 \times 10^{11}$ galaxies in the Cosmos (Christopher J. 2016)

We would have the equivalent of $2.46 \times 10^{22}$ to $10^{23}$ stars in the Universe.

This value is not far from the order of magnitude calculated by expression (6) of $8.64 \times 10^{22}$ suns.

The current estimate of the mass of the observable universe, obtained by other means, is $10^{53}$ $\mathrm{kg}$ (Abhay Deshpande. 2019), not far from of the one of our calculations $1.73 \times 10^{53} \mathrm{~kg}$.

\section{THE COSMOS IN PLANCK TIME}

Using the formula for mass in expression (6), we calculate the amount of mass that could be created in the so-called Planck time. More decimals are used in order to achieve a higher precision.

$$
\mathrm{c}=299792458 \mathrm{~m} \mathrm{~s}^{-1} ; \mathrm{G}=6.67408 \times 10^{-11} \mathrm{~m}^{3} \mathrm{~kg}^{-1} \mathrm{~s}^{-2}
$$

In the Planck time, $\mathrm{T}_{\mathrm{P}}=5.39106 \times 10^{-44} \mathrm{~s}$

$$
\mathrm{M}_{\mathrm{P}}=\mathrm{c}^{3} \mathrm{G}^{-1} \mathrm{~T}_{\mathrm{P}} ; \quad \mathrm{M}_{\mathrm{P}}=21.7643081 \times 10^{-9} \mathrm{~kg}
$$

That is, 21.7643 micrograms, which coincides with the Planck mass.

We can say that the Planck Mass " $\mathrm{M}_{\mathrm{P}}$ " was the mass of the Cosmos when its age was the Planck Time " $\mathrm{T}_{\mathrm{P}}$ ".

Given that mass is proportional to time, this allows us to affirm that the growth rate of the mass of the Cosmos is one Planck mass for each unit of Planck time elapsed.

\section{THE UNIVERSAL CONSTANT "@"}

Equation (6) quantified the growth of the mass of the Cosmos and its value at any point in the evolution of the Cosmos.

However, the reason for this creation of matter is not clear.

In exchange for what is this mass being created?

To support this understanding, we introduce a new universal constant.

We consider that there are four interactions in nature and that at the beginning, they were all connected.

Close to the Planck time, that is, at approximately $10^{-43}$ seconds after the start of everything, the first of these interactions, the Force of Gravity, became independent.

At approximately $10^{-37}$ seconds, the Strong Nuclear Force became independent. 
Finally, at $10^{-12}$ seconds, the Weak Nuclear Force was separated from the Electromagnetic Force.

In this way, it turns out that the electromagnetic force, which is responsible for light, is a consequence of the last separation between interactions.

If we admit that the velocity of propagation of the gravitational fields, to which we assign the symbol "@", is identical to the speed of light in a vacuum "c" (e.g., Abbott B.P. 2017) and assuming that in the cosmic origin, the gravitational fields pre-existed the electromagnetic fields, we must admit that "@" is the cause and "c" is a consequence, so the propagation velocity of the gravitational fields "@" must be considered to be the true universal constant in substitution of "c".

Although "c" and "@" have quantitatively identical values, they are very different conceptually, and we advise that the new constant should be introduced in deductions and calculations.

These ideas were explained by Pavía (Pavía F. 2004) who argued that the appropriate equation for energy should be:

$$
\mathrm{E}=\mathrm{m} @^{2}
$$

\section{THE EQUATION OF THE MASS OF THE COSMOS}

If in equation (6), we substitute "c" for "@", we obtain the final form of the equation sought, that is the equation of the mass of the Cosmos:

$$
\mathrm{M}=@^{3} \mathrm{G}^{-1} \mathrm{~T}
$$

The mass "M" is a function of two universal constants (in this case, both constants are related to gravitational fields) and a single variable, the time " $\mathrm{T}$ ".

In expression (8), it is evident that the gravitational field is responsible for the creation of the mass.

The expression ${ }^{3} \mathrm{G}^{-1}$ is responsible for the creation of mass from the Planck Time to the present and will continue to be so in the future.

Therefore, the creation of mass is the counterpart of the expansion of the Gravitational Field.

Equation (8) suggests that the formation of mass occurs as a by-product of the continuous increase of the gravitational field, which generates space.

Considering expression (7), the equation equivalent to (8) expressed in energy is

$$
\mathrm{E}=@ \mathrm{G}^{5} \mathrm{G}^{-1} \mathrm{~T}
$$

\section{THE SUBSTITUTION OF "c" BY "@"}

We have seen how the substitution of "c" by "@" has allowed a better interpretation of expression (6) when obtaining equation (8).

We must also remember that close to the Planck Time, the electromagnetic interaction was not decoupled, so there was no "c", which strengthens our proposal to generalize the substitution of "c" with "@".

Therefore, we should express the Planck units as 


$$
\mathrm{M}_{\mathrm{P}}=\sqrt{\hbar @ / G} \quad ; \quad \mathrm{T}_{\mathrm{P}}=\sqrt{\hbar G / @^{5}} \quad ; \quad \mathrm{L}_{\mathrm{P}}=\sqrt{\hbar G / @^{3}}
$$

From them, we directly obtain the following: $\quad M_{P}=@^{3} \mathrm{G}^{-1} \mathrm{~T}_{\mathrm{P}}$

Likewise, the Planck Energy is given by $\quad E_{P}=@{ }^{5} G^{-1} T_{P}$

\section{CONSERVATION OF ENERGY AND $M=@^{3} \mathrm{G}^{-1} \mathrm{~T}$}

The principle of energy conservation requires a first adaptation as a result of Einstein's formulation of the equation of energy as a function of the rest mass, $\mathrm{E}=\mathrm{mc}^{2}$ [Stachel John 2001]; from this first moment, the binomial "mass-energy" must be considered as the magnitude that remains constant.

The equation $\mathrm{M}=@^{3} \mathrm{G}^{-1} \mathrm{~T}$, where "M" represents the continuous increase of mass-energy, forces us to reconsider for a second time the law of energy conservation. Now, we find ourselves not with a conservative system, but with an evolutionary system, in which the massenergy increases without end.

$$
\mathrm{E} / @^{2}+\mathrm{M}=@^{3} \mathrm{G}^{-1} \mathrm{~T}
$$

For the cited principle to conform to the conservative aspect, we must express it according to the following equation in a homogeneous way, and referred to energy yields

$$
\mathrm{E}+\mathrm{M} @^{2}-@^{5} \mathrm{G}^{-1} \mathrm{~T}=0
$$

The totality of the energy, mass and 'effect of gravitational fields' of the Cosmos remains equal to zero, regardless of the elapsed time.

\section{THE TOTAL ENERGY OF THE COSMOS IS ZERO}

The gravitational potential energy "P.E." of a set of masses inside a sphere of radius " $R$ " with a total mass "M" is given by P.E. $=-\mathrm{GM}^{2} / \mathrm{R}$

We present the formula $\mathrm{M}=@^{3} \mathrm{G}^{-1} \mathrm{~T}$, which shows that the mass of the Cosmos grows proportionally with time. This expression is consistent with a view of the Cosmos as a gravitational sphere with a radius " $R$ " that corresponds to the distance travelled at the propagation speed of the gravitational fields "@" in a time "T", that is $\mathrm{R}=@ \mathrm{~T}$, wherein the mass and energy are given by expression $\mathrm{M}=\mathrm{Q}^{3} \mathrm{G}^{-1} \mathrm{~T}$.

-Energy of the rest mass:

$$
\mathrm{E}=@^{5} \mathrm{G}^{-1} \mathrm{~T}
$$

- Gravitational potential energy: Applying equations P.E. $=-\mathrm{GM}^{2} / \mathrm{R}$

$$
\text { yields }
$$

$$
\text { P.E. }=-@{ }^{5} \mathrm{G}^{-1} \mathrm{~T}
$$

We observe that the gravitational potential energy of the Cosmos has a negative sign and grows in absolute value with time.

We can see that the two expressions are identical except for the opposite signs, i.e., the sum of the energy corresponding to the mass-energy created in a time "T" (as given by our formula) and the potential energy of the mass of the Cosmos with radius $\mathrm{R}=@ \mathrm{~T}$ is equal to zero. 
We next re-formulate the problem in terms of Planck time. We do not consider the formula for the mass of the Cosmos but instead Planck units are directly used as follows:

$$
\mathrm{M}_{\mathrm{P}}=\sqrt{\hbar @ /_{G}} ; \quad \mathrm{T}_{\mathrm{P}}=\sqrt{\hbar G / @^{5}} ; \quad \mathrm{L}_{\mathrm{P}}=\sqrt{\hbar G / @^{3}} .
$$

The energy equivalent to the Planck mass is $\mathrm{E}_{\mathrm{P}}=\mathrm{M}_{\mathrm{P}} @^{2} ; \quad \mathrm{E}_{\mathrm{P}}=\sqrt{\hbar^{0^{5}} / G}$

The gravitational potential energy in Planck time for a Planck mass within a sphere with a radius of the Planck length (that is, not within a cube) is

$$
\text { P.E.TP }=-\mathrm{GM}_{\mathrm{P}}{ }^{2} / \mathrm{L}_{\mathrm{P}} ; \quad \text { P.E.TP }=-\mathrm{G} \frac{\hbar @ /{ }_{G}}{\sqrt{\hbar G / @^{3}}} ; \quad \text { P.E.TP }=-\sqrt{\hbar \varrho^{5} / G}
$$

The Planck units we obtain for the Planck time show that the energy equivalent to the Planck mass has the same absolute value as the gravitational potential energy but with the opposite sign. Therefore, the sum of these two energies is null.

These simple deductions lead to the conclusion that from an energetic perspective, the Cosmos was born out of nothing, grew into nothing, is nothing and will remain nothing.

Our generation of our entire Cosmos only required a quantum leap, out of which gravity emerged with a propagation speed and time.

The Cosmos evolved in a Planck time " $\mathrm{T}_{\mathrm{P}}$ ", with an initial mass equal to the Planck mass " $\mathrm{M}_{\mathrm{P}}$ " over the space of a sphere with a radius of the Planck length " $\mathrm{L}_{\mathrm{P}}$ ". The radius of this sphere has grown simultaneously and proportionally with the mass-energy. The gravitational potential energy has also grown over time with the same value as the mass-energy, but with a negative sign, resulting in a null net energy.

Our analysis leads to very interesting conclusions.

-The obtained results validate the deduced formula $\quad M=@{ }^{3} \mathrm{G}^{-1} \mathrm{~T}$

-The Planck volume should be considered for a sphere of radius $L_{P}$ and not for a cube of side $\mathrm{L}_{\mathrm{P}}$, as Planck considered and is typically used to obtain the Planck density.

-The obtained results support our view that inherent inconsistencies invalidate the Big Bang hypothesis.

-The results provide a new and clear vision of a Cosmos that was born out of nothing and, in energetic terms, is nothing.

\section{DISCREPANCIES WITH THE STEADY-STATE THEORY}

Given that mass is continuously being created, the cited formula (8) suggests that we are returning to the steady-state theory, as defended by Fred Hoyle, among others, in the middle of the 20th century. These cosmologists claimed that the Cosmos was invariant and that the decrease in its density caused by expansion, was compensated for by the continuous creation of mass.

Despite this apparent similarity to our proposal, there are two facts that completely differentiate our theory from the ideas of Hoyle and the followers of his theory: 
- Our theory is consistent with an equation deduced mathematically from physical formulas. Whereas Hoyle and his followers defended their theory based solely on the idea of keeping the density of the Cosmos constant.

- According to Hoyle, the created matter compensates for the decrease in density as a consequence of expansion. According to our formula, mass grows proportionally with time, while volume grows as the cube of time. Therefore, the density of the Cosmos decreases as a quadratic function of time, not fulfilling the requirements of the steady-state theory.

\section{CONCLUSION}

Equation $M=@^{3} \mathrm{G}^{-1} \mathrm{~T}(8)$ breaks all our schemes.

We affirmed that matter produces gravitational fields. We have now deduced that gravitational fields produce mass (matter).

According to equation (8), the expansion of the Gravitational Field is a counterpart of the creation of mass.

Formula (8) shows us how the immense Cosmos is created with a pattern equivalent to a Planck mass for each unit of Planck Time, that is, the mass of 200000 suns per second.

Our generation of our entire Cosmos only required a quantum leap, out of which gravity emerged with a propagation speed and time.

These deductions lead to the conclusion that from an energetic perspective, the Cosmos was born out of nothing, grew into nothing, is nothing and will remain nothing.

The simplicity, structure and beauty of expression (8) confirm the correct substitution of "c" with "@".

We think that equation (8) and the constant "@" will open a new and interesting field of research and study related to that which is very large and to the very essence of matter.

\section{ACKNOWLEDGMENTS}

We deeply appreciate José Antonio Morales Lladosa and Kevin Alabarta Játiva, for their dedication and good advice that have allowed successive improvements in the text, argumentation, style and presentation. They have ensured that the text has a clear common thread from the beginning to the end.

Likewise, we also thank María García Boronat and Vicente Puig Climent, for their work in translating this document into English and its various preparatory versions.

\section{APPENDIX A}

\section{VARIOUS METHODS TO OBTAIN $\mathbf{M}=@^{3} \mathbf{G}^{-1} \mathbf{T}$}

In this paper we have obtained the equation $\mathbf{M}={ }^{3}{ }^{\mathbf{G}} \cdot \mathbf{T}$, which is of great relevance to the knowledge of the birth and evolution of the Cosmos. Moreover, this expression has in fact been calculated using five different ways. This shows how it is hidden within many physical and nature laws: 
- The first one has been obtained from the two Newton's equations, using the Planck Units in order to get the dimensionless constant "k".

- The second one is a consequence of the expression obtained from the Planck Units, $\mathbf{M}_{\mathrm{p}}={ }^{3} \mathbf{G}^{3} \mathbf{T}_{\mathrm{p}}$, which is used to demonstrate that the value of " $\mathrm{k}$ " is the unit. If we multiply both sides in such equation by a positive integer "N", we obtain the following formula:

$$
\mathbf{N} \mathrm{M}_{\mathrm{P}}=@^{3} \mathrm{G}^{-1} \mathbf{N T}_{\mathrm{P}}
$$

Which clearly states that the mass grows proportionally with the elapsed "quantum of time "or "Plank time".

-In the third one, we assume that the Force of Gravity, was the first interaction that became independent, with its universal constant "G", and its velocity of propagation “@”. Along with the instant in which the time " $\mathrm{T}$ " appears.

Following a similar procedure to the one used by Planck in order to get the "Planck system of units", we will now consider that the fundamental dimensions can be expressed in terms of two universal constants, “G” and “@”, and a variable, time “T”.

We will try to obtain such universal dimensions, universal length $\mathbf{L}_{\mathbf{u}}$, universal mass $\mathbf{M}_{\mathbf{u}}$, and universal time $\mathbf{T}_{\mathbf{u}}$ in terms of @, G, T. In order to do so, we will use the powerful Dimensional Analysis.

The dimensional expressions of such constants, and the time variable, are the following:

$$
[@]=\left[L^{-1}\right] ; \quad[G]=\left[L^{3} M^{-1} T^{-2}\right] ; \quad[T]=[T]
$$

(1)- To express the universal length $\mathbf{L}_{\mathbf{u}}$, in terms of the two constants of gravity and time, we propose the following equation:

$$
\mathbf{L}_{\mathbf{u}}=@ \alpha \mathbf{G}^{\beta} \mathbf{T} \gamma
$$

Matching the exponents:

(L) $1=\alpha+3 \beta+0$

(M) $0=0 \quad-\beta+0$

(T) $\quad 0=-\alpha-2 \beta+\gamma$

With this three equations:

$\alpha=1 ; \beta=0 ; \gamma=1$

and finally, we obtain the expression of "Universal Length":

$$
\mathbf{L}_{\mathbf{u}}=@ \mathbf{T}
$$

(2)- To obtain the universal mass $\mathbf{M}_{\mathbf{u}}$, in terms the two constants of gravity and time, we propose the following equation: 


$$
\mathbf{M}_{\mathbf{u}}=@^{\alpha} \mathbf{G}^{\beta} \mathbf{T}^{\gamma}
$$

Matching the exponents:

$$
\begin{array}{ll}
\text { (L) } 0=\alpha+3 \beta+0 \\
\text { (M) } 1=0 \quad-\beta+0 \\
\text { (T) } 0=-\alpha-2 \beta+\gamma
\end{array}
$$

With this three equations:

$\alpha=3 ; \beta=-1 ; \gamma=1$

which results in the following expression for the Universal Mass:

$$
\mathbf{M}_{\mathbf{u}}=@^{3} \mathbf{G}^{-1} \mathbf{T}
$$

Such expression, coincides with the one that we defined as "Mass of the Cosmos".

(3)- In order to get the universal time $\mathbf{T}_{\mathbf{u}}$, in terms the two constants of gravity and time, we propose the following equation:

$$
\mathbf{T}_{\mathbf{u}}=@^{\alpha} \mathbf{G}^{\beta} \mathbf{T} \gamma
$$

Matching the exponents:

$$
\begin{aligned}
& \text { (L) } 0=\alpha+3 \beta+0 \\
& \text { (M) } 0=0 \quad-\beta+0 \\
& \text { (T) } 1=-\alpha-2 \beta+\gamma
\end{aligned}
$$

With this three equations:

$$
\alpha=0 ; \beta=0 ; \gamma=1
$$

And finally we obtain the following expression for the Universal Time: $\mathbf{T}_{\mathbf{u}}=\mathbf{T}$

-The fourth one, can be calculated from the Cosmos potential gravitational energy. Using the Planck units, expressed in terms of @:

$$
\mathbf{M}_{\mathrm{P}}=\sqrt{\hbar @ / G} \quad ; \quad \mathbf{T}_{\mathrm{P}}=\sqrt{\hbar G / @ 5} \quad ; \quad \mathbf{L}_{\mathrm{P}}=\sqrt{\hbar G / @^{3}}
$$

In the Planck Time: we could consider the Cosmos as a sphere with a radius of the Planck Length " $\mathbf{L}_{\mathbf{P}}$ ", equivalent to the Planck Time multiplied by the propagation velocity of gravity, @: $\quad \mathbf{L}_{\mathbf{P}}=@ \mathbf{T}_{\mathbf{P}}$

The equivalent energy of the Planck Mass is: $\quad \mathbf{E}_{\mathbf{P}}=\mathbf{M}_{\mathbf{P}} @^{\mathbf{2}} ; \quad \mathbf{E}_{\mathbf{P}}=\sqrt{\hbar^{\varrho^{5}} / \boldsymbol{G}}$

Since the potential gravitational energy "P.E." inside a sphere of radius " $R$ " and total mass "M" is: $\quad$ P.E. $=\mathbf{- G M} \mathbf{~}^{2} \mathbf{R}$ 
We obtain:

$$
\text { P.E.TP }=-\mathbf{G} \mathbf{M}_{\mathbf{P}}{ }^{2} / \mathbf{L}_{\mathbf{P}} ; \quad \text { P.E.TP }=-G \frac{\hbar @ / G}{\sqrt{\hbar G / @^{3}}} ; \quad \text { P.E.TP }=-\sqrt{\hbar @^{5} / G}
$$

The potential gravitational energy in the Planck Time, has the same value with opposite sign, as the equivalent energy of the Planck Mass. In consequence, the total energy of the Cosmos in the Planck time is null.

Nowadays: if the total energy of the system in the Planck time was null, why shouldn't it still be null nowadays? This hypothesis has been stated by several cosmologists, although it has not been proved.

And similarly to what we have seen in the Planck time, it could be assumed that currently, the radius of the Cosmos sphere must be $\mathbf{R}=$ @ $\mathbf{T}$, and the corresponding energy of the mass of the Cosmos should be identical to the potential gravitational energy of any mass with a negative sign, so that the total energy is null.

- The equivalent energy "E" of the mass of the Cosmos "M" is: $\quad \mathbf{E}=\mathbf{M} @{ }^{2}$

- The potential gravitational energy "P.E." of the Cosmos in order that the total energy is null, should be:

$$
\begin{aligned}
& \text { P.E. }=-\mathbf{E} \\
& \text { P.E. }=-\mathbf{G ~} \mathbf{M}^{2} / \mathbf{R} ; \quad \mathrm{R}=@ \mathrm{~T} \quad ; \quad \text { P.E. }=-\mathbf{G} \mathbf{M}^{2} / @ \mathbf{T} \\
& \text { - G } \mathbf{M}^{2} / @ \mathbf{T}=-\mathrm{M}^{2} \quad ; \quad M=@^{3} \mathbf{G}^{-1} \mathbf{T}
\end{aligned}
$$

In order that the total energy of the Cosmos is null, the mass of the Cosmos should be expressed as:

$$
\mathbf{M}=@^{3} \mathbf{G}^{-1} \mathbf{T}
$$

Moreover, if the total energy of the Cosmos is null, the mass/energy can't be constant in time, as it is proposed by the Big Bang Theory, since the gravitational fields keep expanding, and so its potential energy changes with time, as $\mathrm{R}$ is different.

- Finally, the fifth one, can be obtained for the energy equation: $\quad \mathbf{E}=\mathbf{h} \mathbf{v}$

$$
\begin{aligned}
& \mathrm{E}=\mathrm{h} v \\
& {[\mathrm{E}]=\left[\mathrm{h} \mathrm{T}^{-1}\right]} \\
& {\left[\mathrm{M} @^{2}\right]=\left[\mathrm{J} . \mathrm{s} \mathrm{T}^{-1}\right] \quad\left(\mathrm{h}=\mathrm{J} \cdot \mathrm{s}=\text { Joules. second }=\mathrm{F} . \mathrm{L} \cdot \mathrm{T}=\mathrm{G} \mathrm{M}^{2} \mathrm{~L}^{-2} \cdot \mathrm{L} \cdot \mathrm{T}\right)} \\
& {\left[\mathrm{M} @^{2}\right]=\left[\mathrm{G} \mathrm{M}^{2} \mathrm{~L}^{-2} \mathrm{LT} \cdot \mathrm{T}^{-1}\right]} \\
& {\left[@^{2}\right]=\left[\mathrm{GML}^{-1}\right]} \\
& {[\mathrm{M}]=\left[@^{2} \mathrm{G}^{-1} \mathrm{~L}\right] \quad[\mathrm{L}]=[@ \mathrm{~T}]} \\
& {[\mathrm{M}]=\left[@^{2} \mathrm{G}^{-1} @ \mathrm{~T}\right]}
\end{aligned}
$$$$
[\mathbf{M}]=\left[@^{3} \mathbf{G}^{-1} \mathbf{T}\right] \quad ; \quad \mathbf{M}=\mathbf{K} @^{3} \mathbf{G}^{-1} \mathbf{T}
$$ 
The dimensionless constant " $K$ " equals the unit, as it was done in the first case, from the Planck Units and $\quad \mathbf{M}_{\mathbf{P}}=@^{3} \mathbf{G}^{-1} \mathbf{T}_{\mathbf{P}}$.

In conclusion, such diversity of procedures that lead to the deduction of the same expression that represents the growth of the Mass of the Cosmos proportionally with time, $\mathbf{M}=@^{3} \mathbf{G}^{-1} \mathbf{T}$, confirms its legitimacy.

\section{APPENDIX B}

\section{DR. DIMITAR VALEV CALCULATIONS}

Not long ago, after we had prepared our paper, we were surprised as we found the article "ESTIMATIONS OF TOTAL MASS AND DENSITY OF THE OBSERVABLE UNIVERSE BY DIMENSIONAL ANALYSIS" by Dr. Dimitar Valev from the "Bulgarian Academy of Sciences, 2011".

Planck obtained his units of Mass, Length, and Time from the constants $\mathbf{G}, \mathbf{c}$, and $\mathbf{h}$, using Dimensional Analysis and a three equation system. We, similarly, obtained the equation $\mathbf{M}=@^{\mathbf{3}} \mathbf{G}^{-1} \mathbf{T}$, using what we think must have appeared in the Cosmos at the beginning of everything, which is the gravity with its two constants( "G" and "@"), and time "T". That has been one of the five ways in which we have calculated the Equation for the Mass of the Cosmos.

In 2011, Dr. Dimitar Valev followed a similar procedure, using Dimensional Analysis, to the one used by Planck in order to obtain his units, introducing the constants $\mathbf{c}, \mathbf{G}$ and $\mathbf{H}$. "H" is the Hubble constant, which substitutes the reduced Planck constant " $\mathbf{h}$ ".

The equation $\mathbf{M}=\mathbf{c}^{\mathbf{3}} / \mathbf{G} \mathbf{H}$, is obtained in that way. Such formula is used to obtain the current mass of the Cosmos.

We must consider the fact that the Hubble Constant " $\mathrm{H}$ " is inadequately called "constant", as it varies with time. When it is expressed in homogeneous units, it is simply the inverse of the Cosmos time, $\mathbf{H}=\mathbf{1} / \mathbf{T}$. Consequently, when the Cosmos had half the current time, the value of $\mathrm{H}$ must have been double the current one.

Therefore, the equation $\mathbf{M}=\mathbf{c}^{\mathbf{3}} / \mathbf{G} \mathbf{H}$ is identical to $\mathbf{M}=@^{\mathbf{3}} \mathbf{G}^{-1} \mathbf{T}$

Dimitar doesn't calculate the value of the adimensional constant " $k$ " in the step from Dimensional Analysis to the definitive equation. He simply estimates that it should be close to one. In our case, we indeed demonstrate that its value is really one, using the Planck Units for the calculation.

In a similar way, Dimitar calculates the density of the current Cosmos, and obtains that

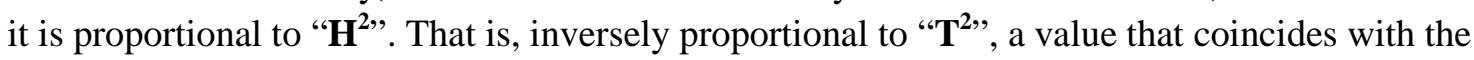
one obtained by us, and consistent with the mass growing proportionally with time, while the volume does so proportionally to the cube of time.

However, we believe that Dimitar just saw the conditions of the present Cosmos in his equations and results, and no more. The progressive meaning contained in his formulas, which 
provides us with the evolutionary aspect of the Cosmos, does not come off from reading his work.

We are under the impression that Dimitar treats the Hubble constant " $\mathrm{H}$ ", as just another constant, stating: "According to recent cosmology, the Hubble constant " $\mathrm{H}$ " slowly decreases with the age of the universe. However, there are indications that other constants, especially the gravitational and fine structure constants, also vary with time. Therefore, the Hubble constant might deserve to be treated on the same level as the other constants".

For all this, we think that Dimitar did not perceive the true and deep meaning of his equations, since their importance is not so much in providing us with the mass and density of the Cosmos at present, but in providing a total paradigm change in relation to the birth and evolution of the Cosmos, thus providing a physical-mathematical basis against the "Big Bang" theory, which lacks of it.

\section{REFERENCES}

-Abbott B.P. et al 2017. Gravitational waves and Gamma-Rays from a binary neutrón Star Merger: GW170817 and GRB 170817A. The Astrophysical Journal Letters V. 848 No 2. -Abhay Deshpande, Rikutaro Yoshida. 2019 june.The Experiment that will probe the deepest recesses of the atom. Scientific American.

-Christopher J. et al 2016. The evolution of galaxy number density at $\mathrm{z}<8$ and its implications. The Astrophysical Journal 830 (2) July.

-Fomin P.I. 1973. Gravitational Instability of vacuum and the cosmological problema.

Bogolyubon Institute for Theoretical Physics, 03680, Kiev, Ukraine. Preprint ITP-73-137.

-Fridman. 1922. Sobre la curvatura del espacio. Zeitschrift für Physik. T. 10.

-Gamow George. 1970. My World Line. Viking, Nueva York

-Guth Alan H. 1999. El Universo inflacionario. Editorial Debate, S.A.

-Hoyle Fred. 1948. A new model for de expanding universe. Monthly Notices of the Royal Astronomical Society.

-Hubble Edwin.1929. A relation between distance and radial velocity among extra galactic nebulae. Procedings of the National Academy of Sciences of the U.S.A.

-Isaacson Walter. 2008. Einstein. Su vida y su universo. Random House Mondadori.

-Lemaître Georges. 1927. A homogeneous universe of constant mass and increasing radius accounting for the radial velocity of extra-galactic nebulae. Annales de la Société scientifique de Bruxelles. 1927.

-Lawrence M. Kraus. 2012. A Universe from Nothing. Free Press, Simon \& Schuster.

-Lynds Peter. 2007. On a finite universe with no beginning or end. arXiv:physics/0612053v3

(physics.gen-ph).

-Maggie Masetti. 2015. How many stars in the Milky Way? NASA blueshift.

-MartínezV.J. et al. 2005. Astronomía fundamental. Universitat de València.

-Pavía F. 2004. mc ${ }^{2}$ versus m@2 ${ }^{2}$ La constante universal "c" en cuestión. HUYGENS No 49, https://www.astrosafor.net/Huygens/2004/49/Cosmologia.htm

-Sanchez del Rio et al. 1997. Física cuántica. Ed. Pirámide Madrid

-Stachel John. 2001. Einstein 1905: Un año milagroso. Editorial Crítica, S.L.

-Tryon Edward P. 1973. Is the Universe a vacuum fluctuation? Nature Vol. 246 December 14.

-Valev Dimitar. 2011. Estimations of total mass and density of the observable universe by

dimensional analysis. Bulgarian Academy of Sciences.

-Vilenkin Alexander. 1982. Creation of Universes from Nothing. Physics Letters 117B

-Weinberg Steven. 1978. Los tres primeros minutos del Universo. Alianza Universidad.

-WMAP 2012. Team receives the Gruber Cosmology Prize, data and results. 